\title{
Coordination and Simplexity in Modelling Air Traffic Control as a Service: The Case of NAV Portugal
}

\author{
Pedro Antunes and Jocelyn Cranefield \\ School of Information Management, Victoria University of Wellington, Wellington, New \\ Zealand
}

\begin{abstract}
This study analyses a unique, revelatory case of service modelling in a complex organisation providing Air Traffic Control. The study analyses broad modelling activities, including information elicitation, analysis and organisation, undertaken by a small team internal to the organisation that so far has spent about 2.400 person/hours of effort in the project. The study follows a qualitative approach in the interpretivist tradition based on interviews and document analysis. The study analyses the project framework, modelling notations, data collection, collaboration, modelling activities, and project outputs. The findings are interpreted in light of two theoretical lenses: coordination and simplexity. The study suggests that simplexity is beneficial for structuring the modelling of complex, knowledge-based services. A pattern was identified combining an initial step promoting simplicity and establishing communication with the stakeholders, followed by a second step acquiring complexity of understanding. Considering the mechanisms defined by coordination theory (flow, share and fit), the study suggests a predominance of the fit mechanism in modelling knowledge-based services. The paper contributes to a better understanding of the challenges of modelling work through cognitive and knowledgebased lenses, and identifies possible strategies to overcome these challenges. The paper also contributes to the emergent literature on simplexity by applying that particular lens to work modelling.
\end{abstract}

Keywords: Service Modelling, Modelling Task, Air Traffic Control, Coordination, Simplexity.

\section{Introduction}

This study analyses a project that modelled Air Traffic Control (ATC) services at a holistic, allof-system level. The project was developed by NAV, which is the Portuguese ATC service provider. The main goal of the project was to ensure compliance with Eurocontrol's European Safety Assessment Regulatory Requirement (ESARR) regarding risk assessment and mitigation. Eurocontrol coordinates ATC across Europe. The main goal of this study was to understand the nature of the modelling task undertaken by the project, which provided a unique, revelatory case of modelling in terms of its criticality, scale and complexity.

This project was an interesting target for research for several reasons. One is that the modelling task was centred on a complex organisation providing a complex, critical service. Lessons taken from these types of organisations and services can, and probably should be, taken into consideration when developing similar projects for less demanding contexts. To give a notion of the complexity involved, the project has spent approximately four years in development and 2.400 person/hours of effort, of which 500 focussed on validation only (Santos 2014). 
Another reason for selection of this case is the nature of the modelling task itself. Modelling in this project was centred on a service that is still predominantly based on humans, even though heavily based on sophisticated technology. Modelling cognitive and knowledge-based functions is a challenge in itself, because capturing information that lives in people's minds and making something useful with that information at the organisational level are both very difficult. The fact that the ATC model resulting from the NAV project is currently being used gives a strong indication that the project had found the right balance between feasibility and utility, which deserves further inquiry. The model is being used for multiple purposes including regulatory compliance, safety analysis, training, knowledge management, and communication, with additional developments under way towards simulation. Furthermore, the model is also being deployed in other ATC organisations.

Finally, another incentive for analysing this project is that the whole project was undertaken by a small team within the organisation itself without support from external consultants, expert modellers and researchers. This is in itself unusual and it is therefore worth investigating the endogenous factors that led to success.

The study followed a qualitative approach in the interpretivist tradition. It was based on analysis of in-depth interviews with key project team members and document analysis. The focus was placed on understanding how the project team undertook broad modelling activities, including information elicitation, analysis and organisation. In particular, we were interested in understanding the decisions that were made by the team and the underlying reasoning for these decisions.

Semi-structured interviews with key project members were recorded then transcribed to text and coded. A set of project documentation was also examined. The data was analysed in two steps: In the first step, an inductive process was used to identify the major decisions taken by the project participants relating to the project framework, modelling notations, data collection, collaboration and modelling. The second round of analysis involved reflecting on these findings about project decisions in light of existing theory. In order to obtain a higher level view, the obtained qualitative results were reframed and interpreted using two relevant theories: coordination (Crowston 1997) and simplexity (Colville 1994).

The paper contributes to a better understanding of the challenges of modelling work through cognitive and knowledge-based lenses, and identifies possible strategies to overcome these challenges. The paper also contributes to the emergent literature on simplexity by applying that particular lens to work modelling.

The paper is organised as follows. In Section 2, we provide background information about the research, mentioning in particular work/enterprise modelling and comparable research studies on modelling. The following section provides details about the study approach. Section 4 provides qualitative insights from our first analytic step, which was centred on interview data and internal documents, while in Section 5 we present the results from the second analytic step, which used theoretical and conceptual frameworks to derive further qualitative insights. Finally, we conclude the paper with a summary of the findings and some discussion points. 


\section{Research Background}

ATC systems are responsible for managing flight operations on the ground and in flight, with the goal of preventing collisions and organizing the flow of aircraft (Carrozza et al. 2013). Despite the use of sophisticated information systems and ongoing technological advances in ATC, human decision-makers are known to play a critical role in safety assurance: The ATC environment is one of high potential risk, in which workers must continually determine priorities while taking into account many simultaneous contextual criteria (Gómez et al. 2016). It is therefore important to engage in modelling of ATC work so as to understand the nature of interactions (human-to-human and human-machine) and decision points and identify the precise areas to target in safety analysis and risk management. Modelling ATC is also of value to inform training and knowledge management. However, modelling of a work setting that is as complex as ATC is a significant and potentially daunting task. Understanding how to successfully undertake such a complex modelling exercise is therefore itself an area of interest. We now consider the issues of modelling and its prior application in ATC studies.

The first issue to address in positioning this study is clarifying what type of modelling we are studying. In the literature the term modelling is characterised by considerable flexibility in meaning. For instance, in research in the ATC context alone, modelling may refer to automation (Rungta et al. 2016), simulation (Jafer et al. 2016), software modelling (Qu et al. 2015), traffic management (Gardi et al. 2015), and/or cognition (Niessen, Eyferth, and Bierwagen 1999). In this study, we adopted a service perspective. This particular perspective emphasises that 1) services are achieved by the interaction between humans and infrastructure under a protocol; and 2) service systems address the coordination, planning and control of humans and infrastructure (Wang et al. 2016). This two-part definition has a strong fit with the ATC context since ATC is strongly dependent on the interaction of humans and technology, and requires complex, swift and flexible coordination and collaboration between them.

Furthermore, the service perspective seems more neutral than other perspectives like business process management (Van der Aalst 2013), which is partial towards a process view, and enterprise modelling (Sandkuhl et al. 2015), which is centred on infrastructure. While services combine people and infrastructure, they do not suggest the predominance of one or the other.

The second issue to consider is that we are focussing on a human activity: the practice of creating a model. In this case, the practice of creating a model for the ATC service. Such activity comprises data elicitation, analysis, modelling using a notation, and model validation. This viewpoint excludes existing research on computational aspects of modelling, e.g. algorithms, mathematical properties, variables, etc. (Samà et al. 2016). The specific knowledge area we are targeting is modelling practice, which is related to the broader concept of design practice (Lehmann et al. 2015) and the more specific concept of process modelling (Bandara and Rosemann 2005).

Our search for literature on the modelling practice of ATC systems from a service perspective brought many results around the topic but we identified no prior studies on this specific intersection of topics. The only prior study we are aware of concerning ATC modelling from a service perspective was published in 1995 (Van Rensburg and Zwemstra 1995). This article presents modelling of workload conditions at different ATC positions but does not provide many insights 
on the actual modelling process. However, we can identify several studies with significant affinities with our goals, outlined below.

Agent-based modelling (Bosse et al. 2013, Bongiorno et al. 2013). Even though agent-based modelling is centred on simulation and therefore unrelated with our research focus, agent systems can describe ATC services at the conceptual level of detail, providing a overall picture of the interconnectivity of the involved actors (Bosse et al. 2013).

Task Analysis (TA) has been applied to ATC (Inoue et al. 2015, Paterno, Santoro, and Tahmassebi 1998). TA has its origins in the Human Computer Interaction field where it has been extensively used to analyse and model how users interact with computer interfaces. TA is based on the observation of users in action. However, the focus is on individual users and micro-tasks, not on the whole service provision.

Cognitive Task Analysis (CTA) has been applied to ATC in several studies (Seamster et al. 1993, Corver and Aneziris 2015, Zachary, Ryder, and Hicinbothom 1998). CTA has its origins in TA, though with a stronger focus on cognitive issues such as attention and situation awareness. It usually starts with interviews with subject matter experts to decompose a task. Later on, auditing is applied to identify the difficult cognitive portions of the task and to seek explanations about why they are difficult (Militello and Hutton 1998). However, CTA shows the same limitation of TA. It can only be applied to specific scenarios, not to whole systems (Zachary, Ryder, and Hicinbothom 1998). For instance, CTA has been used to analyse the use of flight strips by controllers (Corver and Aneziris 2015). The primary reason for the limitation is that the cognitive, task-oriented dimension of the analytic framework cannot scale to the whole organisation.

Cognitive Work Analysis (CWA) has also been applied to ATC (Ahlstrom 2005). Cognitive Work Analysis was created to design large-scale socio-technical systems (Vicente 1999). This approach uses a collection of modelling notations addressing various dimensions of the problem, including the design of the functional work structure, the decomposition of work, and modelling the cognitive decisions involved in accomplishing work. CWA has the capacity to model work at the organisational level, but we are not aware of the application of this method at that scale. Studies have been carried out at a much smaller scale, such as modelling the use of weather displays by controllers (Ahlstrom 2005), and at an intermediate scale, such as modelling the work environment of a control room (Möhlenbrink, Oberheid, and Werther 2008). Perhaps a reason for not using the method to model whole organisations is that it is research intensive, requiring expertise that cannot be easily found inside most organisations.

There are also studies in which Distributed Cognition (DC) has been applied to ATC (Walker et al. 2010, Nilsson et al. 2012). DC theory (Hutchins 1995) emphasises that knowledge is distributed across individual humans and physical environments. The modelling of organisational activities according to this theoretical lens usually leads towards the identification of task and knowledge networks. This seems particularly relevant in the ATC context because the service has to be supported by a network of actors, including multiple controllers in the control room and tower, pilots, and other providers of critical information. The DC approach is primarily centred on identifying design issues by gaining qualitative insights about collaborative work. Schematic models have been developed, for instance describing a control room (Nilsson et al. 2012), but 
again not covering the whole ATC service. Furthermore, because of the nature of the adopted theoretical lens, modelling tends to be performed at a very high level of abstraction.

We thus reiterate the interest in further studying the modelling of ATC from a service perspective. This study provides insights on such a project, with the added significance that the modelling has been carried out entirely by an ATC organisation, without the aid of researchers or other external entities.

\section{Research Approach}

Three key members of the NAV project team were invited to participate in in-depth, semistructured interviews. Those interviewed were the project lead and the modellers involved in the two modelling stages described later on. The interviews were conducted remotely and each one lasted for approximately one hour. Participants were encouraged to share their experiences of the project, with an emphasis in understanding their perspectives and experiences, and the nature and reasons of decisions made. The researcher's understanding of current practices and issues in modelling enabled responsiveness to issues as they arose, with participants being asked more probing questions when known problems and unique practices and processes were being discussed. The interviews were semi-structured, aiming to elicit understanding of the project team members' experiences and understandings. Individual team members were encouraged to share stories that illustrated their experiences, the challenges faced, and the ways in which barriers typical to modelling projects were overcome. The second course of data was a set of documented project outputs including internal technical reports. Our access to these outputs allowed us to identify aspects that were unique to the project, which in turn helped us to focus and fine-tune the interview approach.

The credibility of findings obtained in a study that uses naturalistic inquiry, such as used in this study, are increased through conducting a member check (Lincoln and Guba 1985, 1986). A member check is an analogue of the positivist construct of internal validity that involves, i.e. "soliciting reactions of respondents to the investigator's reconstruction of what he or she has been told or otherwise found out and to the constructions offered by other respondents or sources" (Lincoln and Guba 1985, p.19). In this study, we sent the transcripts/translations of the interviews to the participants and collected clarifications and corrections, which were important because of the specificity of the domain. We also sent our analysis to the study participants and requested feedback regarding how we captured what happened with the project. This induced the participants' reflection about the project and generated many comments, concerns, new viewpoints, and even conflicts that were then addressed and integrated in this study.

In this study credibility was also ensured through triangulation of data sources (interviews and documents) and cross-checking of coding between the researchers.

Because this was a unique, revelatory case, it was also important to consider the criteria of transferability of findings (Lincoln and Guba's analogue of external validity). This was obtained by "thick description" (Lincoln and Guba 1985) in the narrative reporting of findings. Further, we employed the theoretical lenses of coordination theory and simplexity in our final analysis, creating findings at a suitably abstract level for transfer and further investigation. 


\section{$4 \quad$ Initial Analysis}

In the first stage of analysis we began by reading and annotating the transcribed interviews, noting and discussing the key activities, challenges, decisions, and reasons for decisions, reported by participants. We then developed, assigned, merged and revised codes in an inductive and recursive process, aiming to identify the key categories of project-related decisions that had been involved in developing a usable all-of-system ATC model. This analysis resulted in the identification of five thematic categories relating to critical areas of project decisions and activities: (1) the project framework, (2) data collection, (3) modelling notations, (4) collaboration with organizational members, and (5) modelling.

\subsection{Project framework}

The team gave great consideration to the project framework, which had to align the project goals set by the organisation with the selection of modelling notation, adoption of a data collection method and definition of the project outputs. Regarding goals, the project trigger were regulatory requirements enforcing that "any change [in the ATC system] is evaluated to ensure it is safe and that it contributes to the improvement of safety". So the focus was primarily on risk management. Furthermore, the set goals put more emphasis on understanding than developing, which in turn explains why some typical aspects of system modelling were not considered, e.g. data and physical models.

Even though ATC is highly regulated, details of the critical information flows within the organisation were not known by NAV with sufficient precision prior to the project. One participant noted that, "in spite of everybody, for years, having being doing the same thing, exactly the same functions, the notion of what is in reality necessary and how critical each system is, is not really developed". This concern with engineering precision, which may be particular to ATC organisations, is also important to understand the motivations behind the project and the approach that was adopted. The fact that the team had strong backgrounds in methodological rigour and objectivity also shaped the project towards engineering precision (all team members had qualifications in engineering and mathematics).

The process-centric view of modelling fostered by the Business Process Management trend (Biazzo 2000) is currently highly influential (Harmon and Wolf 2014). It is therefore notable that the NAV project was instead largely influenced by a functional view of the organisation. The project team strongly regarded the ATC service as a continuous function. A team member noted: "in our business area, what is important is business continuity" and "we need to constantly supply something: in this case airplanes that have a separation [from any obstacle, including each other]". Even though the purpose of ATC is to safely bring each airplane to ground and from ground to air, expressing these goals in terms of spatial separation is interesting, as it diverts focus to the ensemble instead of the individual units.

However, the team also understood well the concepts of processes and activities. In their view, activities were associated with discrete events related to each airplane from the moment it arrives in the air space until it leaves. Nonetheless, the supplied service was seen as the continuous and simultaneous processing and separation of multiple airplanes. Controllers have to deal with 
multiple airplanes at the same time, and equipment must also do the same. When specifically asked about the differences between the functional and process views, one of the participants referred to the service they provide as being more fluid and therefore more suited to a functional view.

The team did not completely reject the process view, but considered that it was secondary: "We are thinking about that, [we need] to understand the sequences of functions that spring an activity, but that $[\ldots]$ will be considered at a later stage". And they also linked process modelling to optimisation, emphasising they were instead interested on service provision and safety: "If we want to increase performance or reduce costs in certain activities, then we may have to map the activities, which has not been done yet".

The activity centric development of systems was predominant before the 90's but has been succeeded by other approaches such as object-orientation and process-orientation (Chen, Doumeingts, and Vernadat 2008). The participants recognized the anomaly of their success being based around an approach that is widely seen as having significant limitations (Dorador and Young 2000). One team member noted, "what was funny is that I researched new models and even tried to explain them to other people, but always ended up using this one. Because even if it is old, it doesn't matter, it works". Thus it may be the case that such a functional view was not a bias induced by the team but instead an inherent structural property of the organization.

Considerations about precision and detail led the team to early on consider how they would deal with functional decomposition. They decided to organise the project in two phases. One phase addressed the top-level, abstract description of the ATC system, which existing regulations (Commission 2011) designate as "functional system", and the team more informally designate as "[top level] system description". The other phase comprised the lower-level details, which the team called the "system architecture" or the "[whole] model".

The first phase was intended to provide "an abstraction perceptible by everybody" and at the same time to "have this general view but not to lose information". The second phase would provide functional decomposition, until a function could be assigned either to equipment or people.

\subsection{Data collection}

The data collection process was organised by the NAV project team in two stages, interspersed with modelling and validation events. Both stages employed interviews with a range of individuals who were involved in the ATC system.

The first stage of interviews involved 14 people belonging to operational areas, such as traffic management, air space management and operational room management. The capacity to provide global insights about the system activities and, at the same time, be early adopters and key promoters of the model among their peers, were the fundamental criteria adopted by the team to select the participants.

To structure the interviews, the team used a script that started with an explanation of the project goals and the necessary functions, always followed by an initial question: "what do you do that contributes to air traffic safety?". An important goal associated with this question was to understand "the level of abstraction that the person was able to achieve". Then, the discussion was calibrated to elicit information about the activities done by the individual, what each individual 
would need to accomplish an activity in terms of technical support and information provided by systems and people, and whom their activities were serving.

During the interviews, while talking with the subjects, the team would elaborate graphical representations of the discussed activities and information flows using boxes and arrows, "while moving all that information in front of the person, so that the person could see". These informal representations were done on a whiteboard using the minimum possible number of symbols that would require explanations. The focus was on identifying the inputs and outputs of the particular functions done by the individual.

After the interviewees were satisfied with the representations, the team would formalize the representations using IDEF0 to describe functions and information flows and BPMN to describe cognitive activities. These formalizations were done offline. As described by a team member: "after they explained to us what they did, we created a graphical representation using a sequence of boxes in which we showed the activities and the information that flowed between them, systematising what we were told. We transformed a novel into a sequence of tasks". (Note the fluidity between the notions of function and activity.)

After all the stage-one interviews, the team elaborated the top-level system description. This was then validated by the people who had participated in the interviews. Furthermore, many other people from internal and external organisations validated this top-level system description.

In the second stage of the NAV project, further individual interviews were guided to gather additional details about the activities. The scope was expanded to technical support functions such as visualisation support, navigation support, and communications support.

More than 50 individuals were involved in the second stage, including a few that had participated in the first stage. The structure of the interviews was changed to tackle activity decomposition, identification of data inputs and outputs, enablers, equipment, and the authorisations necessary to perform specific activities.

This allowed modelling of the ATC system in increasing levels of detail. That is, the overall project started from the bottom, moved up, and then moved down again, in a "bottom-up-down" pattern.

The team explicitly avoided having either a limit or a rigid number of levels of detail. The number of levels was actually determined after "many hours talking with many people". A team member noted that, "There are functions where going down one level allows understanding what is critical. But there are other more complex functions". The only stop criteria for going down into further levels of detail was being able to assign a function to either a person with a specific authorisation, or to a specific equipment.

As previously noted, given the system complexity, the high-level system description could only emerge after the interviews done in the first-stage of the project. A complete view of the system did not exist at the beginning and could not be developed along with the data collection. It could only be assembled after the first stage was complete. As explained by a team member, to build the first iteration of the top-level system description "[I] closed myself alone in a room for 3 hours looking at an empty whiteboard". 
One factor that perhaps contributed to the lack of a global view during a significant part of the project was the strong compartmentalisation of functions. As noted by a team member, "everything is so compartmentalised that people do not have a notion of the impact they have in the whole operation". Another member noted that during meetings "no one gave an overall view; all of them only gave us partial views".

\subsection{Modelling notations}

The team tested several modelling notations early on in the project. The main criteria adopted to evaluate the alternatives were freedom and ease of use. The former concerned the capacity to move between different levels, adopting top-down and bottom-up perspectives and at the same time keeping the capacity to "relate all the information and check if everything is right". Ease of use was noted as being fundamental to successful working with models during the interviews, because the notation would have to be simple and non-intrusive.

The team reported experimenting using BPMN (Chinosi and Trombetta 2012) to model the whole ATC system. However, this approach did not work. The explanation was that BPMN had fared badly in relationship to interviews: "in the interviews we understood what each worker did; what we didn't understand, and this was why we had to give up on BPMN, was what the decision criteria were". In particular, the team regarded the use of gateways by BPMN as a problem, because they suggest a certain type of interaction with the subject experts that complicated data collection in interviews. One team member also noted "when languages become complex - and nowadays they are very complex - they are not good to communicate".

The team ended up adopting a combination of two modelling notations in phase one and a single notation in phase two.

Regarding phase one, the team adopted IDEF0 and BPMN. IDEF0 was used to represent functions and information flows (Dorador and Young 2000), while BPMN was used to model people's behaviour. In phase two, we could strictly say that no visual notation was used, since the model only exists as a graphical representation in a computer, although partial IDEF0 diagrams can be automatically generated from this. BPMN did not have any role in the second stage, as people's behaviours were represented in the same graph, which could then generate partial IDEF0 notations.

In practice, the team tailored IDEF0 and BPMN to their specific needs. Regarding IDEF0, they used the notation to: 1) provide an overview of activities and information flows at a very general level of detail, e.g. not discriminating between the types of flows; and 2) exhaustively list all information flows pertaining to one single activity. Type 1 was used to describe the functional system and type 2 was used to automatically generate the functional architecture. Furthermore, the team only used top-level IDEF0 diagrams with the typical box syntax, avoiding further complications such as decompositions.

Considering BPMN, very few notational elements were used. Pools, lanes, events, message flows, and artefacts were not used; and only two types of gateways were used. The team used BPMN to document two types of cognitive behaviour: 1) a sequence of cognitive activities from start to finish, e.g. conflict detection involves handling change, checking, finding conflicts and 
maintaining situation awareness; and 2) a cyclic/continuous cognitive activity, e.g. manage traffic, which cycles between situation awareness, conflict detection and a set of possible actions like avoiding collisions and giving instructions to aircraft.

\subsection{Collaboration}

One notable aspect of the project was the high level of collaboration reached between the team and the different participants and departments. Collaboration was reported to have occurred at multiple levels. One concerned collaboration between the team and individual interviewees. During the first-stage interviews, the interviewer had to work with the interviewee in front of the whiteboard to be able to abstract what was said. According to the team, this collaboration exercise was helped by the absence of two common communication barriers: language and modelling notation.

Regarding language, it was noted that the team had thorough knowledge of the organisation and especially of the specific language used in the ATC domain. As noted by one team member, "I've been working in this area since 1996 and so I have some experience about what they do, and what they are trying to explain". Another team member noted "if we do not 'get' the jargon we cannot understand what they say".

A further enabler of collaboration was that no specific notation was used during the interviews. The interviewers only used universally understood words, boxes and arrows.

The lack of modelling notation during the stage-one interviews, and the simplicity of the highlevel system description during the stage-two interviews, contributed to a decrease in communication barriers. As noted by a team member: "I used the minimum [notations] so that everybody could have the same understanding and vision". Also, the team focussed on simple concepts, such as inputs and outputs, before introducing more complex concepts such as controls and mechanisms.

The team observed a willingness from the participants in externalising knowledge: "it was funny that when we asked people what they do - they really liked to talk about it". No resistance to describing work was reported. However, some effort had been necessary to link individual activities together. The people interviewed performed their individual part of the ATC work, but typically did not know how their contribution was linked to others' contributions.

The team recognised that the participants had very different views about the work, and tried to combine their specific goals with the need to preserve such different views. One aspect that contributed to maintain the different views was that the participants were kept involved during the different stages of the project, including validation, and were also kept aware of the high-level system description. The team observed that the participants, after being shown the high-level system description, would easily squeeze in their functions. The high-level system description increased the participants' awareness of how their individual work fitted into the larger ATC system. It was noted, however, that this collaborative behaviour was part of the organisations' ingrained safety culture.

The team also reported that the participants "were really interested in the abstraction of what they do" and "felt good when they understood what they do". One example given was the way in 
which controllers discussed how they maintain situation awareness: "they were very interested in understanding how they build situational awareness. Because they do not know [how it happens], as they do it in a very automatic way".

It was noted, however, that those participants who were most open to collaborate in understanding their work were the ones most aware of their own value, more autonomous and with more control over their decisions. The people who felt they had less importance in the chain of work had more difficulties in collaborating.

The only conflicts faced by the team were related to the second stage of data gathering, where more precise details about activities have been gathered. The conflicts were related to functions that the participants were informally delegating to others, which created grey areas of responsibility. In these cases, specific people within the organisation had to be confronted to understand the actual responsibilities; a process described by one project participant as "playing ping-pong". Despite this challenge, organisational consensus had reportedly always been achieved.

\subsection{Modelling}

When asked to retrospectively analyse the modelling decisions they have made, the team reported very few problems. In particular, the decision to focus modelling on functions instead of processes, the adoption of a simplified version of IDEF0, and the combination of a bottom-up approach to data elicitation with a high-level system description, were all considered very successful.

One problem that was found was integrating the various views of the ATC system; for example, the airplane, the air traffic controller, the many other people supporting the controller, and the equipment. As noted by a team member, "it was very difficult to abstract in [such] a way that our objective was not lost and we could have all these views". As already noted, the adopted approach consisted of having a high-level system description that could be easily visualised and explained to every people involved in the system. However, the lower-level model did not have a visual counterpart. Any time the lower-level model had to be discussed with someone, a specific local view had to be generated using a software tool set developed by the team. The localised views have just the core functions in which a specific participant is involved and link to immediate adjacent functions, but nothing else.

The major problem faced by the team was related to modelling the role of support functions, which are usually performed by technology. As noted by a team member, "when I was trying to link functions with information, I was always missing the dependency that exists with a support function, auxiliary, which in reality represents all the technical component".

An example given by a team member to illustrate the problem concerned situation awareness. This function was part of the manage traffic function, which was one of the nine functions represented in the high-level system description ${ }^{1}$. The situation awareness function required a support function to let controllers set up alarms to alleviate workload. However, adding a link from the situation awareness function to the alarm function seemed inadequate to the team, since every

\footnotetext{
${ }^{1}$ In later developments, the situation awareness function was actually removed from the high-level system description, because of difficulties establishing proper inputs and outputs.
} 
other function would also require the same type of link to many other support functions. That is, auxiliary functions are so ingrained with the other functions that the team found it very difficult to model their specific relationships.

The team ended up aggregating all support functions in one single function, named technical support. This function took a central position in the high-level system description and was linked to every other function in the model. It was also very comprehensive, having 37 inputs and 60 outputs.

Still related to technical support, a team member observed that it was very difficult to model technical support because, unlike human functions who have people to explain them, "I do not have equipment to talk with me".

\subsection{Project Outputs}

The project generated two sets of outputs. One was the functional system, a document with a surprisingly simple collection of models for such a complex organisation. The top model had 9 functions and 11 relationships. Of the 9 functions, only three required decompositions into a second level of detail.

Though these decompositions were also simple. One used a BPMN model with 6 activities. Another used one IDEF0 model with 9 functions. Only the third decomposition, which concerned traffic management, required several models. Traffic management was described with a set of 5 BPMN models. Of these, the most complex one had 11 activities, 3 XOR-splits and 1 AND-split.

The second project output was significantly more complex. It consisted of the functional architecture, which integrated all functions, described at different levels of detail, and all information flows established between functions. One team member noted that some functions were decomposed in 9 levels of detail and the whole functional architecture has about 70 nodes (decompositions and atomic functions), 1200-1300 flows, 500 technical roles, and about 15 human roles. To illustrate the point, a function named "monitor" had 12 inputs, 21 outputs, 3 mechanisms, and 23 control relationships.

The functional architecture does not exist on paper and cannot be visualised in its entirety. Only partial views can be generated. The team developed a software tool set to generate localised views, which show functions and information flows relevant to a particular actor. Furthermore, the team also developed tools to check model consistency and to automatically generate documentation.

\section{Further Analysis}

In interpreting the study findings at a higher level we draw on two theoretical lenses that have synergy with experiences and themes reported above: coordination theory (Crowston, 1997) and the emergent concept of simplexity (Colville, Brown, and Pye 2012, Rego 2010).

According to the well-known theory of coordination proposed by Crowston (1997), we can find three types of dependencies in coordinated work: task-task, task-resource and resource-resource; also designated as flow, share and fit (Malone et al. 1999). Flow relationships occur where the inputs of some activities depend on the outputs of other activities preceding them. Share relationships concern concurrent access to resources, while fit relationships arise when various activities collectively contribute to generate an output. 
Flow (or task-task) relationships are the typical focus of process-oriented modelling approaches. As noted in our analysis above, the project team rejected this view to model the ATC system. The main reason was that they could not see the actors accomplishing discrete goals and performing discrete activities. For instance, a controller does not control a single airplane. Instead, the controller is responsible for maintaining "separation" between multiple airplanes. In that strict perspective, there is no input waiting for an output. The controller also depends on the provision of aeronautical information on a regular basis, but not on a task basis.

Perhaps another way of looking at the issue is that, for this particular organisation, the actors' performance is neither planned nor controlled on a piece-meal basis. This is especially true for controllers. Our initial analysis indicated that controllers were aware of their value, were autonomous and had control over their decisions. As a consequence, they seem to be managed according to their capabilities but not their individual activities.

The share (or task-resource) view also does not seem adequate to the ATC system because in this particular organisation functions do not compete for resources. For instance, "traffic management" is not competing with "response to anomalies" or any other function for aeronautical information. Quite the contrary, "traffic management" may have to collaborate with "response to anomalies" when some problem is detected by either a controller or an aircraft.

Perhaps that explains why the project team did not look at the task-resource dependencies involved in, for example, "traffic management". However, they did model the communication flows between tasks that required/provided critical information resources, such as in the case of "traffic management" and "response to anomalies", which have mutual dependencies.

So, according to Crowston's coordination theory, the remaining dependency that could be considered is the fit (or resource-resource) dependency. In this view, we could say that the highlevel system description identifies a set of functions that must fit together to produce the single output of an ATC system: separation. The fit dependency seems to better reflect what was predominantly modelled by the team: information flows. Especially in the low-level models developed by the team, each function was accompanied by a large number of inputs, outputs, controls and mechanisms, which elucidated how the different flows fitted together at certain points.

The team's decision to avoid detailing which actors, either humans or machines, participated in the high-level system description also seems consistent with the fit view. Humans and machines were modelled in a way that emphasises simultaneous agency. For instance, controllers use alarms to alleviate workload.

More recent studies of coordination suggest expanding the notion beyond interdependencies. In particular, Okhuysen and Bechky (2009) suggest looking at three conditions that preclude coordination: accountability, predictability and common understanding. Accountability concerns making clear where the responsibilities of interdependent parts lie, while predictability allows anticipating how the parties perform their tasks, and common understanding provides a shared view of the whole task.

Regarding our data from this lens, we can identify the elements that contributed to these three conditions and how they were organised by the team. Accountability was only provided by the low-level models, where every function was related to a particular actor, which could be either a 
human or a machine. On the other side of the coin, removing accountability from the high-level models created problems with the support functions but increased ease of use.

Some degree of predictability can be found in the models through the specification of inputs, outputs, mechanisms and control relationships. However, these relationships do not define the precise timing of tasks and events, and neither do they reflect the existence of a work plan, routine or process. The adopted approach seems suitable to a work environment based on trust, independence and knowledge-based work.

Common understanding was perhaps the strongest condition found in the project. The team built the high-level and low-level models from the ground up with support from more than 50 people involved in the ATC system. The top-level models were specifically developed to deliver common understanding and according to our data that goal was very successful.

The complexity of the working environment seems to be reflected in the degree of common understanding developed by the team. On the one hand, the high-level models provided a simplified but holistic view of the entire ATC system, while on the other hand, the low-level models provided very complex views, so complex that they could only be partially generated for specific actors.

A second set of insights arising from the initial data analysis is related to the concept of simplexity that has been recently proposed in the research literature (Colville, Brown, and Pye 2012, Rego 2010). The purpose of simplexity is to make sense of complex organisations through the combination of simplicity and complexity.

The project organisation and the collection of models generated by the ATC project illustrate how simple and complex views of reality can be combined. The functional system provided a highlevel view of the whole ATC system and was surprisingly simple, even though it did not omit any important relationship. As noted by the team, such simplicity was instrumental in communicating with the other members of the organisation with the purpose of understanding the complexity of work. On the other side of the coin, the functional architecture, which was significantly more complex, so much that it only existed in the computer, contributed to the primary goal of the project, which was to improve safety. Without the former, the later would not have been be possible; and without the later the former would not have been useful.

Furthermore, the concept of simplexity also addresses the challenges of bringing together multiple views about the ATC system. As noted by the team, the organisation did not have a comprehensive view of the ATC system, since work was compartmentalised. Key members like the controllers were not aware of the specific contributions of others to their own work.

Using the simplexity lens, we could summarise the modelling approach described in this paper as 1) building simplicity first and complexity later; and 2) communicating simplicity to acquire complexity of understanding.

One final issue that should be considered is the organisational culture necessary to adopt such a modelling approach. First, we have to consider that it would be difficult, if not impossible, to build such a model in a less stable organisation. The model has been under development since 2012 and during that period the service has not changed significantly. Only the support technology has 
changed, which does not significantly impact the model. Many organisations would not be able to provide such stability.

Second, the team had a strong cultural orientation towards a formal, systematic approach in understanding work, which can go into great levels of detail and precision. The team's background in engineering and mathematics contributed to such orientation.

Third, the team's two-stage approach fostered internal commitment to the project. The early interviews with key personnel in the development of the high-level system description generated momentum, while the more extensive interviews done in the second stage contributed to buy-in and a sense of co-design.

The curiosity of key personnel in understanding the way they work also contributed positively to the project. Finally, the reported levels of collaboration in model development also suggest a strong organisational culture supporting the project.

In Figure 1 we summarise the key elements that emerged from the study.

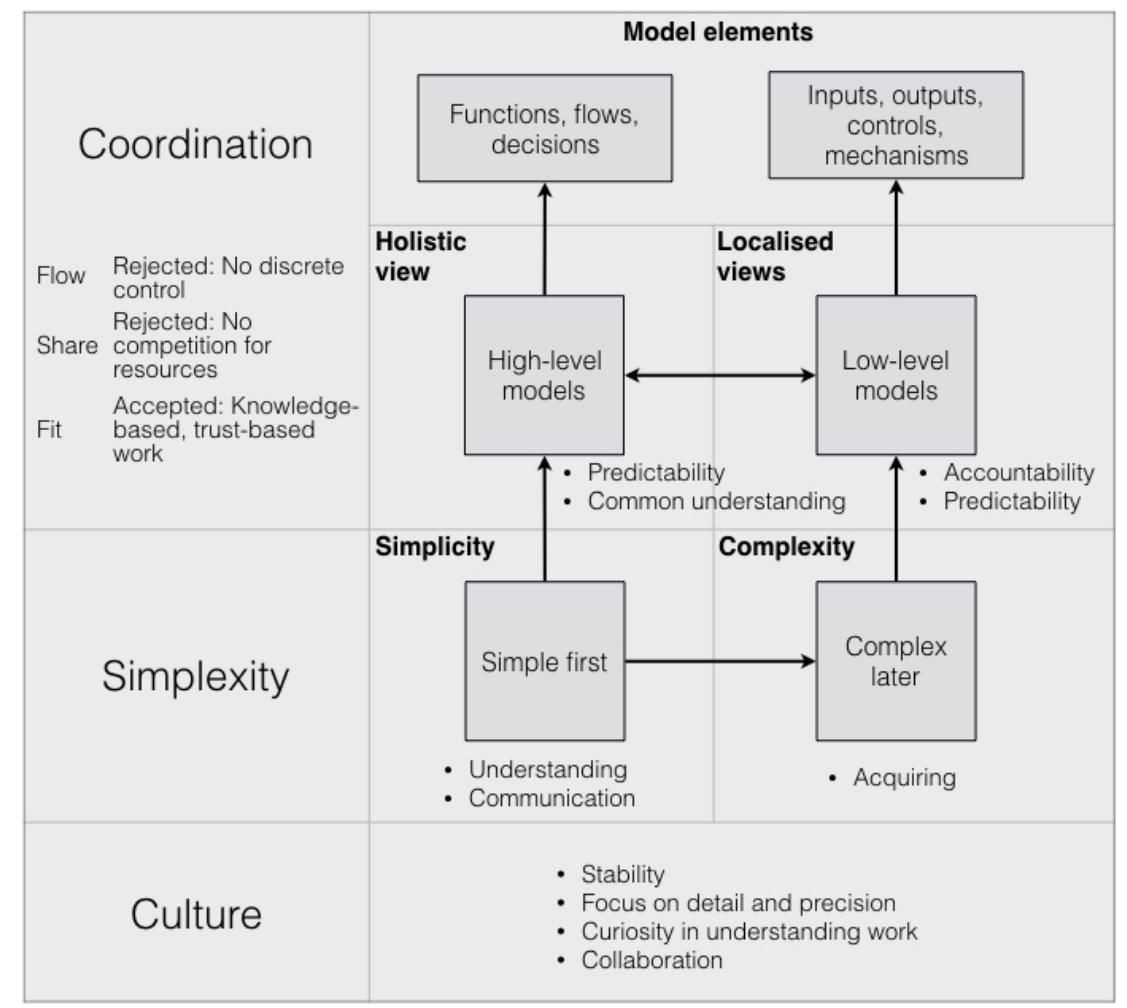

Figure 1 - Summary of key elements that emerged from data analysis 


\section{Discussion and Conclusions}

Simplexity theory suggests that, to make sense of complex organisations, one needs to combine complexity of thinking with simplicity of action (Colville, Brown, and Pye 2012). This viewpoint was apparent in the way NAV approached the project. In particular, we observed that NAV divided the project in two phases, the first one adopting a bottom-up approach with the purpose to develop a holistic/simple model and the second phase adopting a top-down approach to develop a detailed/complex model of the ATC service.

This combination of simplicity and complexity created the capacity to simultaneously communicate the model to the stakeholders and to gather knowledge from them, which was necessary to actually build the model. In other words, simplicity allowed moving forward the modelling task, while complexity allowed reaching the goals of having a useful ATC model.

Can this pattern seeking the simple first and complex later in modelling be extrapolated to other areas? To answer this question, we have to analyse other contextual factors framing the researched project. One that emerged during the study was the stakeholders' curiosity in understanding work (especially the controllers). We suggest such curiosity derives from the complexity of work itself, which emphasises knowledge and cognition. The other factor is the organisation's focus on detail and precision, which is derived from the high-risk environment in which it operates. Therefore, we suggest that the observed simplexity pattern to modelling could be extrapolated to organisational areas with knowledge-based workers operating in high-risk environments, which would for instance include healthcare and emergency management.

One key element that stands out from the study is the team's adoption of a functional view of work. Such view has been criticised for several weaknesses, such as being unable to represent sequences of activities and information structures (Dorador and Young 2000). Regarding activities in particular, the functional view is positioned as a step backward when compared with the processcentric view fostered by the Business Process Management trend (Biazzo 2000), which is currently highly influential across multiple businesses (Harmon and Wolf 2014). For instance, a survey across multiple industries reports that $93 \%$ of organisations do business process modelling, and that $76 \%$ of organisations use business processes to communicate (Harmon and Wolf 2011). In the paper, we show how the project team took a different approach and explain why it was successful.

Another interesting topic that emerged in this study was the project team's selection of a modelling notation. To recap, the team adopted IDEF0 after having experimented with BPMN and realising that it would not be adequate to their case. In our analysis, we note that the primary reason was that workers were not controlled based on the tasks they accomplish but instead based on their overall performance. This again reflects the strong cognitive and knowledge bases of the organisation.

We adopted coordination theory to further analyse the problem, noting that coordination has been divided into three types named flow, share and fit. Regarding NAV, we observed that both the flow and share categories would not apply, while fit seemed adequate. Because BPMN is nowadays the predominant notation for enterprise modelling, we may ask: Can we derive any implications from this study regarding BPMN? 
We note that BPMN provides a strong notation for expressing the flow and share types of coordination. More specifically, the flow type is reflected in the semantics of activities and sequence flows (OMG 2011). The share type is reflected in the semantics of data objects and data flows. However, we cannot find any adequate element in BPMN to reflect the fit type. The fit type requires having information flows between activities, but not control flows. Other elements in the BPMN notation support information flows, but they restrict them to actors, in the case of collaborations and choreographies. Therefore, based on this study, we could suggest that the BPMN notation could be extended to include communication flows between activities. Such extension would have allowed the NAV team to use BPMN in modelling the ATC service. We also suggest that such extension would also be useful to model work processes in areas such as healthcare and emergency management where activities are not controlled on a piecemeal basis but instead rely on strong interdependence, trust and collaboration.

All in all, this study provides two contributions to research. This first one is bringing simplexity theory to service modelling. The study suggests that the simplexity lens is adequate to structure the modelling of complex services. The second contribution is suggesting that process modelling should comprehend the three types of coordination suggested by coordination theory: flow, share and fit.

Regarding contributions to practice, the study elucidates how a successful project aimed at modelling a very complex service was conducted, providing insights into the problems, challenges and decisions that were made by the project team.

\section{Acknowledgements}

Special thanks to Paula Santos, Paulo Monteiro, and Maria João for their support to this research. We would also like to thank NAV Portugal for giving us access to their project.

\section{References}

Ahlstrom, U. 2005. "Work domain analysis for air traffic controller weather displays." Journal of safety research 36 (2):159-169.

Bandara, W., and M. Rosemann. 2005. "What Are the Secrets of Successful Process Modelling? Insights From an Australian Case Study." French Journal of Strategic Information Systems 3 (10):47-68.

Biazzo, S. 2000. "Approaches to business process analysis: a review." Business Process Management Journal 6 (2):99-112.

Bongiorno, C., G. Gurtner, F. Lillo, L. Valori, M. Ducci, B. Monechi, and S. Pozzi. 2013. "An agent based model of air traffic management." Proceedings of the SESAR Innovation Days, EUROCONTROL.

Bosse, T., H. Blom, S. Stroeve, and A. Sharpanskykh. 2013. "An Integrated Multi-agent Model for Modelling Hazards within Air Traffic Management." In Proceedings of the 2013 IEEE/WIC/ACM International Joint Conferences on Web Intelligence (WI) and Intelligent Agent Technologies (IAT), 179-186. IEEE Computer Society. 
Carrozza, G., M. Faella, F. Fucci, R. Pietrantuono, and S. Russo. 2013. "Engineering air traffic control systems with a model-driven approach." IEEE software 30 (3):42-48.

Chen, D., G. Doumeingts, and F. Vernadat. 2008. "Architectures for enterprise integration and interoperability: Past, present and future." Computers in industry 59 (7):647-659.

Chinosi, M., and A. Trombetta. 2012. "BPMN: An introduction to the standard." Computer Standards \& Interfaces 34:124-134.

Colville, I. 1994. "Searching for Karl Weick and reviewing the future." Organization 1 (1):218224.

Colville, I., A. Brown, and A. Pye. 2012. "Simplexity: Sensemaking, organizing and storytelling for our time." Human Relations 65 (1):5-15.

Commission, European. 2011. Commission Implementing Regulation No 1035/2011.

Corver, S., and O. Aneziris. 2015. "The impact of controller support tools in enroute air traffic control on cognitive error modes: A comparative analysis in two operational environments." Safety science 71:2-15.

Crowston, K. 1997. "A coordination theory approach to organizational process design." Organization Science 8 (2):157-175.

Dorador, J., and R. Young. 2000. "Application of IDEF0, IDEF3 and UML methodologies in the creation of information models." International Journal of Computer Integrated Manufacturing 13 (5):430-445.

Gardi, A., R. Sabatini, T. Kistan, Y. Lim, and S. Ramasamy. 2015. "4 dimensional trajectory functionalities for air traffic management systems." Integrated Communication, Navigation and Surveillance Conference (ICNS)

Gómez, S., A. Goron, A. Groza, and I. Letia. 2016. "Assuring safety in air traffic control systems with argumentation and model checking." Expert Systems with Applications 44:367-385.

Harmon, P., and C. Wolf. 2011. Business Process Modeling Survey. Business Process Trends

Harmon, P., and C. Wolf. 2014. The State of Business Process Management 2014. Business Process Trends

Hutchins, E. 1995. Cognition in the wild. Cambridge, MA: MIT Press.

Inoue, S., K. Yamazaki, H. Hirako, and T. Sasaki. 2015. "Applying human centered design process for designing air traffic control interfaces." International Conference of Design, User Experience, and Usability.

Jafer, S., B. Chhaya, U. Durak, and T Gerlach. 2016. "Formal Scenario Definition Language for Aviation: Aircraft Landing Case Study." AIAA Modeling and Simulation Technologies Conference.

Lehmann, M., N. Bocken, J. Steingrímsson, and S. Evans. 2015. "Incorporating design thinking into sustainable business modelling." Proceedings of the 2nd International Conference on Sustainable Design and Manufacturing, Seville, Spain.

Lincoln, Y., and E. Guba. 1985. Naturalistic Inquiry. Beverly Hills, California: Sage.

Lincoln, Y., and E. Guba. 1986. "But is it rigorous? Trustworthiness and authenticity in naturalistic evaluation." New directions for program evaluation 30:73-84. 
Malone, T., K. Crowston, J. Lee, B. Pentland, C. Dellarocas, G. Wyner, J. Quimby, C. Osborn, A. Bernstein, G. Herman, and M. Klein. 1999. "Tools for inventing organizations: Toward a handbook of organizational processes." Management Science 45 (3):425-443.

Militello, L., and R. Hutton. 1998. "Applied cognitive task analysis (ACTA): a practitioner's toolkit for understanding cognitive task demands." Ergonomics 41 (11):1618-1641.

Möhlenbrink, C., H. Oberheid, and B. Werther. 2008. "A model based approach to cognitive work analysis and work process design in air traffic control." In Human Factors for assistance and automation, 401-414.

Niessen, C., K. Eyferth, and T. Bierwagen. 1999. "Modelling cognitive processes of experienced air traffic controllers." Ergonomics 42 (11).

Nilsson, M., J. van Laere, T. Susi, and T. Ziemke. 2012. "Information fusion in practice: A distributed cognition perspective on the active role of users." Information fusion 13 (1):6078.

Okhuysen, G., and B. Bechky. 2009. "coordination in organizations: an integrative perspective." The Academy of Management Annals 3 (1):463-502.

OMG. 2011. Business Process Model and Notation (BPMN) Version 2.0. Object Management Group

Paterno, F., C. Santoro, and S. Tahmassebi. 1998. "Formal models for cooperative tasks: concepts and an application for en-route air traffic control." Design, Specification and Verification of Interactive Systems' 98.

Qu, J., R. Wu, J. Yang, and W. Wang. 2015. "Software reliability analysis in air traffic control system." Integrated Communication, Navigation and Surveillance Conference (ICNS).

Rego, A. 2010. "Complexity, simplicity, simplexity." European Management Journal 28 (2):8594.

Rungta, N., E. Mercer, F. Raimondi, B. Krantz, R. Stocker, and A. Wallace. 2016. "Modeling complex air traffic management systems." Proceedings of the 8th International Workshop on Modeling in Software Engineering.

Samà, M., A. D’Ariano, P. D'Ariano, and D. Pacciarelli. 2016. Scheduling models for optimal aircraft traffic control at busy airports: tardiness, priorities, equity and violations considerations Omega.

Sandkuhl, K., A. Smirnov, N. Shilov, and H. Koç. 2015. "Ontology-Driven Enterprise Modelling in Practice: Experiences from Industrial Cases." International Conference on Advanced Information Systems Engineering.

Santos, P. 2014. "Modelling day to day ATM." Safety, Human Performance, System: From Theory to Practice, Lisbon, Portugal.

Seamster, T., R. Redding, J. Cannon, J. Ryder, and J. Purcell. 1993. "Cognitive task analysis of expertise in air traffic control." The International Journal of Aviation Psychology 3 (4):257-283.

Van der Aalst, W. 2013. "Business Process Management: A Comprehensive Survey." ISRN Software Engineering. 
Van Rensburg, A., and N. Zwemstra. 1995. "Implementing IDEF techniques as simulation modelling specifications." Computers \& Industrial Engineering 29 (1):467-471.

Vicente, K. 1999. Cognitive Work Analysis: Toward Safe, Productive, and Healthy ComputerBased Work. Mahwan, NJ: Lawrence Erlbaum Associates, Inc.

Walker, G., N. Stanton, C. Baber, L. Wells, H. Gibson, P. Salmon, and D. Jenkins. 2010. "From ethnography to the EAST method: A tractable approach for representing distributed cognition in Air Traffic Control." Ergonomics 53 (2):184-197.

Wang, J., H. Wang, J. Ding, K. Furuta, T. Kanno, Ip W., and W. Zhang. 2016. "On domain modelling of the service system with its application to enterprise information systems." Enterprise Information Systems 10 (1):1-16.

Zachary, W., J. Ryder, and J. Hicinbothom. 1998. "Cognitive task analysis and modeling of decision making in complex environments." In Making decisions under stress: Implications for individual and team training, 315-344. 